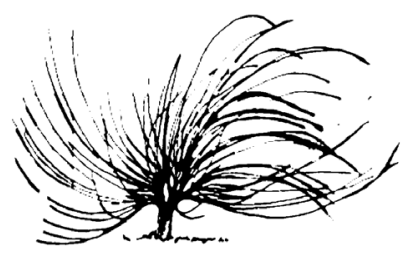

\title{
En busca de la inclusión educativa Una propuesta de aula a partir del Aprendizaje Cooperativo y las TRIC
}

\author{
Rodrigo García Pajares ${ }^{1}$ \\ Universidad de Valladolid \\ España \\ garciapajaresr@gmail.com
}

\begin{abstract}
Resumen
Este trabajo tiene como objetivo mostrar las experiencias surgidas a partir del desarrollo de una propuesta de innovación educativa en un centro urbano de la ciudad de Segovia (España). La finalidad de la propuesta es lograr un modelo de aula inclusivo en el que todos los estudiantes se sientan partícipes del proceso de aprendizaje. Para conseguirlo, hemos decidido unir el modelo pedagógico del Aprendizaje cooperativo (AC), junto al trabajo con las Tecnologías de la relación, la información y la comunicación (TRIC). La razón por la que se combinan ambos elementos es el potencial inclusivo que despiertan por separado, así como el interés por este tipo de prácticas educativas. Con el fin de valorar si la propuesta de aula planteada cumple con los objetivos que nos hemos propuesto, se ha llevado a cabo un estudio de caso con el que se pretende comprobar empíricamente su veracidad. Este se elabora a partir de un análisis cualitativo basado en técnicas de observación. Toda la información recogida nos conduce a la divulgación de unas interesantes conclusiones sobre este campo.
\end{abstract}

Recibido: 9 de diciembre de 2018. Aprobado: 12 de setiembre de 2019.

http://dx.doi.org/10.15359/rep.14-2.8

1 Graduado en Educación Primaria, mención Educación Física por la Universidad de Valladolid. 
Palabras clave: aprendizaje cooperativo, educación inclusiva, innovación educativa, tecnologías de la información y la comunicación, tecnologías de la relación, información y comunicación.

\begin{abstract}
This paper aims to show the experiences arising from the development of a proposal for educational innovation in an urban center of the city of Segovia (Spain). The purpose of the proposal is to achieve an inclusive classroom model in which all students feel part of the learning process. To achieve this, we have decided to unite the pedagogical model of Cooperative Learning (CL) together with the work with Information, Relationship, and Communication Technologies (IRCT). The reason why both elements are combined is the inclusive potential that they arise separately, as well as the interest in this type of educational practices. In order to assess whether this classroom proposal accomplished the stated objectives, a case study was carried out to verify its veracity empirically. This was elaborated from a qualitative analysis based on observation techniques. All the information collected leads us to the disclosure of some interesting conclusions about this field.
\end{abstract}

Keywords: Cooperative Learning, inclusive education, educational innovation, Information and Communication Technologies, Information, Relationship and Communication Technologies

\title{
Introducción
}

ctualmente, existen numerosos aportes que, sin duda, favorecen $\triangle$ el continuo progreso hacia la mejora del panorama educativo, 1 pero debemos hacer una autocrítica y valorar cuáles son los aspectos sobre los que se debe de seguir trabajando. Desde nuestra posición, la falta de inclusión de todo el alumnado en el aula es uno de ellos. Aunque, la ignorancia nos pueda llevar a pensar que todos nuestros estudiantes tienen una participación más o menos equitativa y gozan de las mismas oportunidades de aprender, la realidad es muy distinta. Lo cierto es que en el aula existe una minoría que se hace notar y una mayoría que se manifiesta como silenciosa. Por norma general, el equipo 
docente suele enfocar sus clases hacia esa minoría más destacada y no atiende verdaderamente a la mayoría de su alumnado (Fernández, 2018).

El presente trabajo tiene un carácter innovador al buscar una educación inclusiva que atienda idealmente al $100 \%$ de los estudiantes. Cuando hablamos de una propuesta de innovación no lo hacemos porque la metodología utilizada se trate de algo que surja fruto de nuestra invención, sino más bien se debe a que el objetivo de esta busca paliar un defecto encontrado. El concepto innovación educativa hace referencia a aquella acción de cambio en el marco de la institución educativa en la que se busca efectividad en relación con objetivo propuesto (Monge y Gómez, 2018). Innovar, por tanto, no solo es buscar aquello que consideramos que no está bien para mejorarlo, sino que también implica lograr el objetivo propuesto.

Para nosotros innovar es lograr una educación marcada por su carácter inclusivo, y para conseguirlo nos servimos de la hibridación del modelo de Aprendizaje cooperativo y el trabajo con las TRIC. La unión no resulta sencilla a simple vista, pero sí lo es cuando tomamos como referencia aquellos puntos de unión entre ambos. Para que se entienda mejor explicaremos uno a uno cómo contribuyen al logro de nuestro particular objetivo. Tras ello, mostraremos cuáles son los resultados obtenidos a partir de su unión en la propuesta de intervención educativa que hemos diseñado. De esta manera comprenderemos el verdadero sentido de lo que significa innovación educativa.

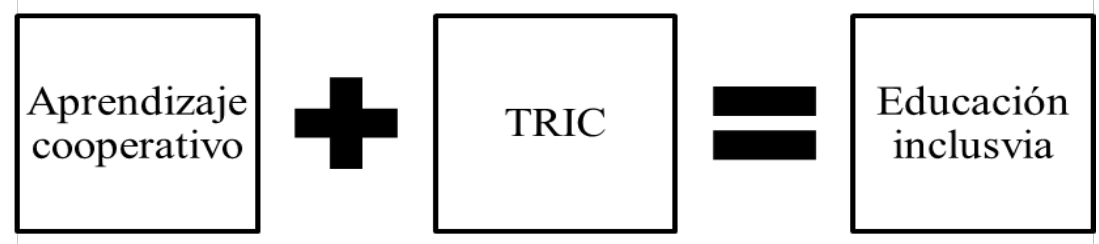

\section{Figura 1. Fórmula en la que se muestra la hibridación del AC y TRIC para obtener una educación inclusiva.}

Nota: elaboración propia.

\section{Antecedentes}

A lo largo de esta propuesta se presenta un híbrido, elaborado a partir del modelo del Aprendizaje cooperativo y el trabajo con las Tecnologías de la relación, de la información y de la comunicación, cuyo 
objetivo es el de lograr una práctica de aula marcada por su carácter inclusivo. En este sentido, se entiende por educación inclusiva el conjunto de prácticas educativas encaminadas, en las cuales todo el alumnado se sienta partícipe del proceso de enseñanza-aprendizaje, independientemente de sus características, necesidades o intereses (Arnáiz, 2003). Tenemos especial interés sobre las aportaciones realizadas en Ainscow (2012), Arnáiz (2003), y Martínez y Gómez (2013), cuyas ideas y principios en torno a su modelo de educación han calado sustancialmente, sobre nuestras prácticas educativas. Se considera oportuno realizar una breve revisión de las diferentes aportaciones de los dos medios que hemos seleccionado para llegar a este modelo.

En primer lugar, empezaremos por el modelo del Aprendizaje cooperativo (AC), el cual consideramos, más que una alternativa, una necesidad educativa. Su génesis se basa en la lógica de que un producto es mejor si se hace en grupo y no por separado. Es la historia de cualquier celebridad que nunca habría logrado llegar a la cima sin el apoyo de otras personas. ¿Qué sería de un piloto de automovilismo sin su equipo, de un presidente sin sus asesores, de un profesor sin sus alumnos...? Esa es la base sobre la que se construye este modelo y que podría resumirse con aquella expresión que decía: "uno para todos y todos para uno". Un lema que llevado a lo académico nos permite la construcción del aprendizaje de forma colectiva, haciendo mucho más enriquecedor el proceso para los que participan en él.

Para Johnson, Johnson y Holubec (1999), el término de aprendizaje cooperativo se define como: "el empleo didáctico de grupos reducidos en los que los alumnos trabajan juntos para maximizar su propio aprendizaje y el de los demás” (p. 5). Posee cinco pilares básicos: la interdependencia positiva, la interacción promotora cara a cara, las habilidades personales, la responsabilidad individual en el trabajo colectivo y el procesamiento grupal (Johnson y Johnson, 2014). Sus elementos constituyentes hacen que se adapte tanto a las asignaturas "troncales" como al resto de específicas, como podría ser la educación física (Velázquez, Fraile y López, 2014); por lo que se torna como un modelo aplicable a cualquier materia del currículo, tal y como lo demuestran los diferentes estudios y trabajos efectuados en torno a esta temática. Entre los que destacamos los realizados en Jonhson et al. (1999), y Slavin y Jonhson (1999). Además, tendremos especialmente en cuenta el trabajo desarrollado en Pujolàs (2012), al tratarse de un 
referente en la utilización de esta metodología con fines inclusivos. En resumidas cuentas, este modelo sugiere un cambio en la dinámica del proceso de enseñanza-aprendizaje tradicional que va en la línea del trabajo con los medios tecnológicos.

Las Tecnologías de la Relación, de la Información y de la Comunicación, abreviado TRIC, es un concepto quizás no muy conocido y que está íntimamente ligado a la era de la digitalización. Las tecnologías han llegado para formar parte de nuestro día a día y para abarcar todos los ámbitos de la sociedad con sus respectivos cambios (Caldevilla, 2010), e influencias desde edades muy tempranas (Osuna, Gil y Cantillo, 2018). La enseñanza, al igual que el resto de contexto social, va incluyendo estos dispositivos electrónicos para beneficiarse de ellos, tanto en espacios analógicos como digitales, favoreciendo de este modo el empoderamiento del alumnado (Osuna y Gil, 2017). De ahí que surjan nuevas denominaciones dentro de nuestro ámbito, como el de las aulas 2.0 (Domingo y Marqués, 2011), la alfabetización digital (Gutiérrez y Tyner, 2012), o el de gamificación (Díaz y Troyano, 2013).

El nombre que reciben dichos elementos es el de Tecnologías de la Información y Comunicación (TIC). El motivo de que sean nombradas de esa forma, y no de otra, es por tener funciones relacionadas con el almacenamiento, procesamiento y transmisión de datos (Rodríguez, 2009). Existen diversos trabajos que nos hablan sobre las innumerables ventajas de dicha inclusión en el contexto educativo, nos gustaría destacar las siguientes: posibilita el aprendizaje, no solo en la escuela, sino que, en cualquier escenario, al acercar la información al individuo (Lozano, 2011), incrementa en cantidad el número de medios y formas de organizar y representar la información sobre la que se trabaja, y permite el desarrollo de capacidades ligadas a la creatividad (García y González, 2006), hace posible la ruptura con las barreras espacio-temporales (Ferro, Martínez, y Otero, 2009), genera autonomía en el alumno, al tener que pensar y actuar de manera individual, aunque también con otros (Kozulin, 2000), y por último, fomenta la sociabilidad (Busquet, Medina y Ballano, 2013). En esta última ventaja podemos observar la precisión que realizan Marta y Gabelas (2016), al presentar el concepto de TRIC. Estas se convierten en uno de los elementos principales del presente trabajo. 


\section{Referentes teóricos}

\section{El aprendizaje cooperativo: un modelo para la inclusión}

En este planteamiento el alumnado pasa a tener una interdependencia positiva con el resto de componentes del grupo que les hace sentir la necesidad de trabajar cooperativamente. Para que esto suceda no solo basta con dividir el aula en grupos reducidos y heterogéneos, también es necesario que el profesorado adopte un rol de guía y favorezca situaciones en las que se precise de la cooperación (Pujolàs, Riera, Pedrogosa y Soldevila, 2005). Existen cinco pilares básicos, los cuales ya han sido mencionados anteriormente. Aunque, para que el modelo de trabajo de aula sea considerado un trabajo cooperativo auténtico debe de existir una participación equitativa y una igualdad de oportunidades de éxito (Pujolás, 2004, 2009). Estos dos son considerados el sexto y séptimo elemento básico del AC (Fernández, 2018), y, como no, los tomamos como aspectos clave para la consecución de nuestro particular fin.

La participación equitativa es símbolo de inclusión. Si existe una paridad a la hora de contabilizar las aportaciones de cada uno de los componentes del grupo, se podría decir que todos tienen una participación activa y, por lo tanto, están incluidos en el aula. Nunca lograremos una equidad absoluta, pero sí se puede conseguir que todas tengan una oportunidad para realizar su aportación. Al sintetizar el formato del aula, de un gran grupo a varios pequeños, favorecemos la inclusión y la participación de la mayoría de los individuos (Jonhson et al., 1999).

Por otro lado, se encuentra la igualdad de oportunidades de éxito, que fundamentamos en una idea de lo más sencilla y completamente entendible: para que el estudiante se sienta integrado dentro de la clase, este debe de sentir que está contribuyendo al logro grupal; por lo tanto, si fomentamos que todos y cada uno de los interactuantes tengan la oportunidad de lograr algún éxito individual, estos se sentirán partícipes de los logros colectivos, llevando implícito el sentimiento de pertenencia al grupo, que es sinónimo de inclusión. Como docentes podemos favorecer que esto ocurra proponiendo actividades que requieran habilidades y capacidades muy distintas, plantear actividades abiertas, incluir contenidos conceptuales, procedimentales y actitudinales o evaluar con flexibilidad (Fernández, 2018).

Estos son los principales motivos por los que consideramos de suma utilidad la aplicación del AC, para conseguir un aprendizaje más 
integrador. Los cinco elementos básicos de este modelo más los dos añadidos, nos ofrecen siete aspectos que facilitan la consecución de una educación inclusiva. Nos mantenemos firmes en la idea de cambio de la realización de actividades de tipo individual, por otras de carácter cooperativo, que hacen del aula un espacio inclusivo para todas aquellas personas que se encuentran en ella (Pujolàs, 2012).

\section{El trabajo mediante las TRIC. Un medio para promover la interactividad y el acercamiento interpersonal}

De entre todas las ventajas surgidas a partir de la implementación de las TIC en el aula, nos suscita especial interés su potencial para fomentar la sociabilidad. Es decir, la forma en la que facilitan situaciones de interacción social entre los participantes en el proceso de enseñanza-aprendizaje. Para centrarnos en esta característica dejamos a un lado el concepto TIC, y nos vamos a referir a ellas con la denominación TRIC (Garrido, Munté y Busquet, 2016). El motivo de este cambio se debe a que estamos considerando que las nuevas tecnologías favorecen espacios o situaciones en las que el alumno se relaciona, interactúa y comunica con sus iguales e incluso con el propio docente (Gil y Marfil, 2018). Estamos diciendo, por tanto, que estos dispositivos favorecen que el sujeto hable, se comunique o simplemente interactúe. De modo que un dispositivo puede ser una herramienta clave para establecer un acercamiento hacia otra persona, por medio de un diálogo vía oral "cara a cara" o también, mediante un soporte digital, a lo que se le conoce por el término interactividad (García y González, 2006).

Las TRIC crean entornos de aprendizaje en los que se potencia sustancialmente la interacción, puesto que, además de facilitar situaciones de relaciones interpersonales, abren una vía más para la comunicación. El alumnado maximiza las posibilidades de relacionarse con sus semejantes y con el profesorado, ya que no solo se pueden comunicar en el tiempo que comparten en el aula, sino también fuera de ella, a través del software social. Ha surgido un nuevo medio para iniciar un intercambio comunicativo que hace tener un contacto permanente en la comunidad de aprendizaje (Calzadilla, 2002), así, el grupo estudiantil amplía sus posibilidades para la relación, y por tanto, para la inclusión, al tener medios para acercarse al resto de individuos y construir comunidad. 


\section{Estrategia metodológica}

El conocimiento teórico de estos ámbitos es el que posibilitará desarrollar una estrategia metodológica que se adapte a nuestros intereses. De modo que, para dar respuesta a la falta de inclusión en los centros escolares, se ha planteado un estudio de caso con el que se pretende conocer empíricamente, si la hibridación del AC junto al trabajo mediante las TRIC da lugar a ambientes de aprendizaje más integradores. La íntima conexión con la realidad que nos aporta este método (Martínez, 2006), se torna como un elemento clave por el que se ha realizado tal elección.

La muestra que ha participado en el estudio es un total de cincuenta alumnos de sexto de Educación Primaria procedentes de un colegio urbano de la capital de Segovia. Los estudiantes, en su mayoría, proceden de núcleos familiares con un contexto socioeconómico de nivel medio y aproximadamente, una cuarta parte de ellos son de procedencia extranjera. El centro escolar ha instaurado varios proyectos en los que se apuesta por la innovación educativa mediante los dispositivos electrónicos. Es destacable este hecho puesto que, gracias a ello, todo el proceso se ha visto favorecido.

Los objetivos que se plantean con este trabajo son los siguientes:

1. Valorar si el trabajo con escolares mediante la unión de las TRIC y del modelo del AC favorecen espacios de aprendizaje marcados por la inclusión.

2. Analizar de forma individual en qué grado las TRIC fomentan la inclusión del alumnado dentro del aula.

Basándonos en estos objetivos, presentamos a continuación la hipótesis del trabajo: una metodología de trabajo de aula en la que se incluya de manera simultánea el modelo de AC y el trabajo con las TRIC nos ofrece unos importantes resultados como es la educación inclusiva.

Para evaluar los fines marcados a través de este estudio nos hemos servido de la metodología cualitativa y, con base en esta, la técnica de rejilla de observación. Hemos establecido una serie de indicadores a observar, los cuales están íntimamente ligados al análisis de los objetivos propuestos con el trabajo. Se ha elegido esta forma de recogida de información, por permitir centrar nuestros esfuerzos de observación 
sobre aspectos concretos y, además, por la riqueza de los datos cualitativos obtenidos que nos lleve a la mejora de la calidad educativa, al partir de una experiencia real de aprendizaje.

\section{Propuesta de intervención}

Para poder llegar hacia unas ideas mucho más fundamentadas se planteó un trabajo de aula dentro de una metodología de trabajo por proyectos, concretamente planteados desde el área de Ciencias Sociales, aunque con un tinte interdisciplinar. Este se convierte en un modelo que representa la idea que queremos transmitir con este escrito. La puesta en práctica con escolares, que se basa en el trabajo cooperativo unido al empleo de las TRIC, tuvo unas fases que han sido seguidas: formación de grupos, asignación de roles, explicación del trabajo por realizar, trabajo por equipos y divulgación del conocimiento (puzzle de Aronson).

1-Formación de grupos, tal y como se recoge en los principios del AC, para garantizar su correcto desarrollo, se organizaron de forma heterogénea (Pujolàs et al., 2005). Para garantizar la heterogeneidad se optó por establecer unos principios que a su vez buscan la inclusión de todo el alumnado:

- Aquellos estudiantes a los que les resulte más complejo interaccionar o relacionarse con el resto de alumnado estuvieron acompañados con un compañero con el que tuviesen una mayor afinidad.

- Los grupos son mixtos y tratamos de que en cada agrupamiento haya el mismo número de estudiantes del sexo femenino que del masculino.

- $\quad$ Se buscó un equilibrio entre el alumnado más capaz para el estudio y los que presentan mayores dificultades.

2-Asignación de roles dentro del grupo, lo idóneo es que cada conjunto esté compuesto por cuatro estudiantes, por lo que hubo el mismo número de roles distintos: el encargado, el ayudante del encargado, el portavoz, el secretario y el encargado del material. Los roles deben de tener unas tareas asignadas que estén claras (Tabla 1), son rotativos y las funciones asignadas a cada uno se tienen que revisar periódicamente (Pujolàs et al., 2005). 


\section{Tabla 1.}

Funciones asignadas a cada uno de los roles desempeñados en el grupo

\begin{tabular}{|c|c|}
\hline Encargado/ayudante del encargado & Portavoz \\
\hline $\begin{array}{l}\text { - Coordina el trabajo de todo el grupo } \\
\text { y se encarga de que todo funcione } \\
\text { correctamente. } \\
\text { Es la persona que más claro tiene } \\
\text { lo que les está pidiendo el profesor. }\end{array}$ & $\begin{array}{l}\text { - Comunica al profesor dificultades } \\
\text { encontradas en el proceso de } \\
\text { aprendizaje. } \\
\text { Si es necesario es la persona que } \\
\text { acude a otro grupo para tratar temas } \\
\text { relacionados con el trabajo. }\end{array}$ \\
\hline Secretario & Encargado del material \\
\hline $\begin{array}{l}\text { - Anota posibles problemas con las } \\
\text { funciones asignadas a cada rol, para } \\
\text { su posterior revisión. } \\
\text { - Sobre él recae la responsabilidad de } \\
\text { que todo el trabajo quede guardado. } \\
\text { Ningún aparato se apaga sin su } \\
\text { consentimiento. }\end{array}$ & $\begin{array}{l}\text { - Es el encargado de cuidar, recoger y } \\
\text { guardar las tablets, así como el resto } \\
\text { de material que se haya utilizado. } \\
\text { - Controla que la zona de trabajo se } \\
\text { encuentre limpia antes, durante y } \\
\text { después de la sesión. }\end{array}$ \\
\hline
\end{tabular}

Nota: elaboración propia, con base en los roles que se utiliza en Pujolàs et al. (2005).

3-Explicación del trabajo a realizar, cada grupo tuvo que elaborar una parte del tema que se ha trabajado, favoreciendo la investigación en la red. En concreto, la temática estaba relacionada con la sociedad del consumo, que es trabajada a partir de una propuesta de intervención de carácter interdisciplinar, en la que se utiliza el juego para tratar la educación para el desarrollo (García, 2018). El docente asignó a cada grupo una parte del tema para desarrollarlo y entre todos se elaboró el conjunto por escrito (Tabla 2).

Tabla 2.

Índice sobre el tema que ha elaborado cada grupo de trabajo para el desarrollo del tema final "La sociedad del consumo"

\begin{tabular}{|l|l|l|l|}
\hline \multicolumn{1}{|c|}{ LA SOCIEDAD DEL CONSUMO } \\
\hline \multicolumn{1}{|c|}{ Grupo 1 } & \multicolumn{1}{|c|}{ Grupo 2 } & \multicolumn{1}{|c|}{ Grupo 3 } & \multicolumn{1}{c|}{ Grupo 4 } \\
\hline EI dinero & El consumo & Las empresas & Los recursos \\
- ¿Qué es el dinero? & - ¿Qué es el & -Tipos de empresas & -Recursos naturales. \\
¿Dónde se utiliza? & consumo? & y sectores & -Recursos \\
- Formas de & -Efectos del & económicos. & económicos. \\
intercambio & consumismo. & - ¿Cómo influyen & - ¿Cómo afecta el \\
utilizadas en el & -El gasto y el & las empresas sobre & consumo sobre los \\
pasado. & ahorro. & el consumo? & recursos? \\
\hline
\end{tabular}

Nota: elaboración propia. 
Cada equipo de trabajo contó con tres tablets y sus cuadernos de aula. A partir de ahí, se recogieron los contenidos que se consideraban necesarios de entre un amplio listado de páginas web recomendadas por el docente. Desde esta perspectiva las tecnologías se proyectan como una fuente para la adquisición del aprendizaje y del conocimiento (Lozano, 2011). La información recogida se redactó finalmente, en un Google Drive conjunto, en el que cada grupo tendrá unas páginas asignadas elaborándose así un producto final accesible para todo el alumnado.

4-Divulgación del conocimiento, siguiendo la técnica del puzzle de Aronson, se procedió al desarrollo de la última parte. Esta consistió en lo siguiente: cada equipo se ha convertido en un experto sobre una parte del tema, es por ese motivo por el cual ahora un integrante de cada grupo se reparte por las mesas para que en cada una haya tan solo un estudiante de cada grupo (Traver y García, 2006). Por turnos uno a uno describe su parte del tema al resto que escuchan y preguntan, en caso de duda, al acabar la explicación. De esta forma pasamos de la colaboración (trabajo por equipos) a la cooperación (compartimos el aprendizaje al resto de grupos). Los alumnos pudieron utilizar la tablet para realizar la exposición de su parte.

\section{Resultados y discusión}

Como ya se apreció nos decantamos por las rejillas de observación, para obtener una información que será la que nos lleve a establecer unos resultados sobre nuestra propuesta. Para realizar el análisis de las rejillas de observación se ha optado por separar, por un lado, la parte referida al AC y por el otro, el de las TRIC, para luego elaborar un análisis final en el que se abarque ambos, desde la visión que nos ofrece su combinación.

\section{Análisis de las rejillas de observación: AC}

Existen dos razones destacables para poder considerar el modelo del AC como una forma de trabajo de aula que favorece la inclusión. Una de ellas ha sido el más que notable aumento de la participación del alumnado que antes apenas realizaba aportaciones al aula, lo que es símbolo de inclusión (Barrio, 2008). Como se puede apreciar en la Tabla 3, en la primera sesión no todas las personas implicadas cumplían las funciones relacionadas con su rol, por lo que había estudiantes, 
cuya aportación al grupo era más bien escasa. Con el desarrollo de las posteriores sesiones este grupo estudiantil ha ido asumiendo el rol asignado y, por lo tanto, ha tenido una implicación dentro del grupo. La asignación de roles es un elemento clave dentro de este modelo y se ha podido comprobar que en el momento en el que cada uno cumple con sus "obligaciones", todos tienen una participación activa y todos están incluidos en el trabajo de aula.

La otra es el equilibrio que se ha formado entre interactuantes "más capaces" para los estudios y aquellos a los que les cuesta más trabajo. Estos últimos están acostumbrados a ver cómo el resto de la clase avanza y ellos se quedan atrás. Al seguir el modelo del AC se ha podido apreciar como las dificultades encontradas se iban resolviendo entre ellos al ver que el trabajo era tarea de todos (interdependencia positiva). Es cierto que al inicio buscaban ayuda en la figura del profesor, pero, poco a poco, ellos mismos eran los que resolvían sus problemas. Este tipo de situaciones nos hacen valorar el potencial inclusivo de lo que se conoce como "el niño tutor". Por tanto, las prácticas de aula inclusivas se logran a partir de la dinamización de los recursos humanos disponibles con el fin de lograr mejoras en la participación y en el aprendizaje (Ainscow, 2012).

\section{Análisis de las rejillas de observación: TRIC}

$\mathrm{Al}$ igual que hemos hecho anteriormente, vamos a destacar los aspectos ligados a las TRIC que más han contribuido a lograr un aula más inclusiva. En primer lugar, nos gustaría valorar las tecnologías como un medio para establecer un acercamiento de carácter social. Dentro de un aula hay alumnado a los que no les resulta fácil iniciar un diálogo con otras personas; esa timidez es la que puede provocar que existan personas menos integradas. Se ha comprobado que, en este caso, las tablets y los contenidos incluidos en ellas, facilitaban el acercamiento de unos con otros, despertando labores de colaboración y ayuda. Este hecho se debe, en parte, a la amplia variedad de habilidades que son necesarias para desempeñar cualquier tipo de función con ellas, lo cual provoca la implicación de varias personas. El colegio público de Ariño (Teruel) es un verdadero ejemplo de cómo utilizar el programa de Escuela 2.0 para lograr un modelo de aula inclusivo (Martínez y Gómez, 2013).

La inclusión de este tipo de estudiantes, también se favorece por medio de la interactividad que nos ofrecen las TRIC. Se ha apreciado 
que algunos interactuantes quedaban para avanzar o acabar sus trabajos fuera del aula y que utilizaban soportes digitales para comunicarse desde sus casas. Para los jóvenes a los que les resulta más costoso entablar una conversación "cara a cara", así como para el resto de ellos, las TRIC son una herramienta que utilizan para conocerse, presentarse y construir su identidad (Garrido et al., 2016).

Tabla 3.

\section{Rejilla de observación número 1}

\section{REJILLA DE OBSERVACIÓN. HIBRIDACIÓN DEL AC Y TRIC}

Proyecto: Hibridación del AC y las TRIC para una educación inclusiva.

Objetivo/s (*):

Observar si el modelo de trabajo en el aula fomenta espacios de aprendizaje inclusivos para el alumnado.

Valorar cómo contribuye cada uno de ellos (AC y TRIC) al logro de nuestro fin. Clase: Ciencias Naturales $6 .^{\circ}$ Primaria

Tema: La sociedad del consumo

Sesión $\mathrm{n} .^{\circ} 1$

Duración de la clase: 1 hora

\begin{tabular}{|l|l|l|l|}
\hline \multicolumn{1}{|c|}{ INDICADOR } & SÍ & NO & \multicolumn{1}{c|}{ OBSERVACIONES } \\
\hline $\begin{array}{l}\text { Los alumnos hablan e intercambian } \\
\text { puntos de vista sobre el tema. } \\
\text { Cohesión de grupo. }\end{array}$ & $\mathrm{X}$ & & $\begin{array}{l}\text { Se puede apreciar como existen } \\
\text { constantes charlas en cada uno de } \\
\text { los grupos. }\end{array}$ \\
\hline $\begin{array}{l}\text { El alumnado solicita la ayuda de } \\
\text { sus compañeros antes que la del } \\
\text { docente. }\end{array}$ & & $\mathrm{X}$ & $\begin{array}{l}\text { Aunque, se observa que se producen } \\
\text { ciertas ayudas entre los propios } \\
\text { compañeros, siguen acudiendo al } \\
\text { profesor de forma instintiva. }\end{array}$ \\
\hline $\begin{array}{l}\text { Cada integrante cumple con su } \\
\text { rol de grupo y mantiene una } \\
\text { interacción continua con el resto } \\
\text { del alumnado. }\end{array}$ & & $\begin{array}{l}\text { De vez en cuando, se tiene que } \\
\text { recordar el rol de cada uno dentro } \\
\text { del equipo. Les cuesta asumir una } \\
\text { función concreta y ceñirse a lo que } \\
\text { se les pide. Aún hay algún caso en el } \\
\text { que la aportación al grupo es escasa. } \\
\text { A pesar de ello, se va reforzando la } \\
\text { idea de los roles y se va asumiendo. }\end{array}$ \\
\hline $\begin{array}{l}\text { Se generan diálogos gracias a } \\
\text { situaciones promovidas por el uso } \\
\text { de la tablet. }\end{array}$ & $\mathrm{X}$ & $\begin{array}{l}\text { La mayoría de los diálogos que se } \\
\text { están produciendo están muy ligados } \\
\text { o son originados a partir de las } \\
\text { tablet, normalmente, el intercambio } \\
\text { de información se produce para la } \\
\text { elección de una u otra página web } \\
\text { para desarrollar su temática. }\end{array}$ \\
\hline
\end{tabular}


Se muestran indicios de haber adelantado trabajo fuera del aula y, por lo tanto, de haber establecido contactos mediante soportes digitales.
Al ser la primera sesión no han establecido contactos de carácter interactivo al no saber aún qué es lo que íbamos a trabajar.

Nota: elaboración propia, con base en los sucesos observados en el aula.

\section{Tabla 4.}

\section{Rejilla de observación número 2}

\section{REJILLA DE OBSERVACIÓN. HIBRIDACIÓN DEL AC Y TRIC}

Proyecto: Hibridación del AC y las TRIC para una educación inclusiva

Objetivo/s: $(*)$

Clase: Ciencias Naturales $6 .^{\circ}$ primaria

Tema: La sociedad del consumo

Sesión $n^{\circ} 2$

Duración de la clase: 1 hora

\begin{tabular}{|l|l|l|l|}
\hline \multicolumn{1}{|c|}{ INDICADOR } & SÍ & NO & \multicolumn{1}{c|}{ OBSERVACIONES } \\
\hline $\begin{array}{l}\text { El alumnado habla e intercambia } \\
\text { puntos de vista sobre el tema. } \\
\text { Cohesión de grupo. }\end{array}$ & $\mathrm{X}$ & & $\begin{array}{l}\text { Se ha detectado cómo gracias a la } \\
\text { formación en grupos estudiantes que } \\
\text { antes no se relacionaban ahora sí lo } \\
\text { hacen. Un ejemplo de ello es una } \\
\text { conversación que se producía en uno } \\
\text { de los grupos al final de la clase, un } \\
\text { alumno preguntaba al otro si tenía } \\
\text { cuenta de alguna red social. }\end{array}$ \\
\hline $\begin{array}{l}\text { El alumnado solicita la ayuda de } \\
\text { sus compañeros antes que la del } \\
\text { docente. }\end{array}$ & & $\begin{array}{l}\text { Son aisladas las situaciones en } \\
\text { las que el alumnado acude al } \\
\text { docente para solicitar ayuda, pero } \\
\text { aún se siguen dando con cierta } \\
\text { normalidad. Su prioridad suele ser } \\
\text { la de acudir al docente primero y } \\
\text { ya después a sus compañeros de } \\
\text { grupo. Pero es destacable que se } \\
\text { dan numerosas ayudas entre los } \\
\text { propios componentes del grupo, } \\
\text { normalmente, son las personas más } \\
\text { dados al estudio los que ayudan al } \\
\text { resto. }\end{array}$ \\
\hline
\end{tabular}




\begin{tabular}{|l|l|l|l|}
\hline $\begin{array}{l}\text { Cada integrante cumple con su } \\
\text { rol de grupo y mantiene una } \\
\text { interacción continua con el resto } \\
\text { del alumnado. }\end{array}$ & $\mathrm{X}$ & & $\begin{array}{l}\text { Entre sus semejantes se recuerdan } \\
\text { las funciones de cada uno. Cuando } \\
\text { hay casos en los que un integrante } \\
\text { no cumple su rol, normalmente, se } \\
\text { le recuerda para que lo haga. De esta } \\
\text { forma, en la mayoría de los grupos } \\
\text { todos sus componentes tienen una } \\
\text { participación activa. }\end{array}$ \\
\hline $\begin{array}{l}\text { Se generan diálogos gracias a } \\
\text { situaciones promovidas por el uso } \\
\text { de la tablet. }\end{array}$ & $\mathrm{X}$ & $\begin{array}{l}\text { Las interacciones se intensifican } \\
\text { al tener que manejar programas } \\
\text { digitales que permitan plasmar la } \\
\text { información que están recogiendo. } \\
\text { Se requieren de diversas habilidades } \\
\text { que pocos estudiantes tienen para } \\
\text { hacerle frente a esto. Por ese motivo, } \\
\text { se pregunta o se charla con otros } \\
\text { compañeros. }\end{array}$ \\
\hline $\begin{array}{l}\text { Se muestran indicios de haber } \\
\text { adelantado trabajo fuera del aula y, } \\
\text { por lo tanto, de haber establecido } \\
\text { contactos mediante soportes } \\
\text { digitales. }\end{array}$ & $\mathrm{X}$ & $\begin{array}{l}\text { Se ha podido observar casos en } \\
\text { los que el alumno sugiere a otro } \\
\text { "hacerlo mejor en casa y mandárselo } \\
\text { por correo electrónico". }\end{array}$ \\
\hline
\end{tabular}

Nota: elaboración propia, con base en los sucesos observados en el aula.

\section{Tabla 5.}

\section{Rejilla de observación número 3}

\section{REJILLA DE OBSERVACIÓN. HIBRIDACIÓN DEL AC Y TRIC}

Proyecto: Hibridación del AC y las TRIC para una educación inclusiva

Objetivo/s: $\left(^{*}\right)$

Clase: Ciencias Naturales $6 .^{\circ}$ primaria

Tema: La sociedad del consumo

Sesión $n .^{\circ} 3$

Duración de la clase: 1 hora

\begin{tabular}{|c|c|c|c|}
\hline INDICADOR & SÍ & NO & OBSERVACIONES \\
\hline $\begin{array}{l}\text { El alumnado habla e intercambia } \\
\text { puntos de vista sobre el tema. } \\
\text { Cohesión de grupo. }\end{array}$ & $\mathrm{X}$ & & $\begin{array}{l}\text { Los componentes de cada grupo } \\
\text { presentan en la mayoría de las } \\
\text { agrupaciones una buena relación } \\
\text { entre sí. Así lo muestran sus } \\
\text { múltiples interacciones. } \\
\text { Durante la técnica del puzzle de } \\
\text { Aronson, se observa un buen clima } \\
\text { de trabajo que ha favorecido las } \\
\text { relaciones entre el alumnado. }\end{array}$ \\
\hline
\end{tabular}




\begin{tabular}{|l|l|l|l|}
\hline $\begin{array}{l}\text { El alumnado solicita la ayuda de } \\
\text { sus compañeros antes que la del } \\
\text { docente. }\end{array}$ & $\mathrm{X}$ & & $\begin{array}{l}\text { El papel del profesor ha pasado a } \\
\text { un segundo plano, son aisladas las } \\
\text { situaciones en las que se requiere } \\
\text { de la ayuda del docente. La mayoría } \\
\text { de los problemas los resuelven ellos } \\
\text { mismos. }\end{array}$ \\
\hline $\begin{array}{l}\text { Cada integrante cumple con su } \\
\text { rol de grupo y mantiene una } \\
\text { interacción continua con el resto } \\
\text { del alumnado. }\end{array}$ & $\mathrm{X}$ & $\begin{array}{l}\text { Los integrantes de cada grupo } \\
\text { han asumido las funciones que } \\
\text { tienen que desempeñar, lo cual, ha } \\
\text { facilitado una participación activa de } \\
\text { todo el alumnado. }\end{array}$ \\
\hline $\begin{array}{l}\text { Se generan diálogos gracias a } \\
\text { situaciones promovidas por el uso } \\
\text { de la tablet. }\end{array}$ & $\mathrm{X}$ & $\begin{array}{l}\text { La tablet o los contenidos que se } \\
\text { encuentran dentro de ella son una } \\
\text { especie de herramienta para "romper } \\
\text { el hielo" iniciar una conversación. } \\
\text { Algún ejemplo de lo que se ha } \\
\text { observado: "qué chulo ide qué } \\
\text { página lo has sacado?", o “cómo lo } \\
\text { has hecho para que te quede así? }\end{array}$ \\
\hline $\begin{array}{l}\text { Se muestran indicios de haber } \\
\text { adelantado trabajo fuera del aula y, } \\
\text { por lo tanto, de haber establecido } \\
\text { contactos mediante soportes } \\
\text { digitales. }\end{array}$ & $\mathrm{X}$ & $\begin{array}{l}\text { Hay claros indicios de que ha } \\
\text { existido trabajo fuera del aula, } \\
\text { ya que varios grupos no habían } \\
\text { concluido su trabajo durante la } \\
\text { sesión pasada. Este hecho implica } \\
\text { que, casi con total seguridad, ha } \\
\text { habido conversaciones mediante } \\
\text { algún soporte digital, para tratar } \\
\text { temas relacionados con su trabajo. }\end{array}$ \\
\hline
\end{tabular}

Nota: elaboración propia, con base en los sucesos observados en el aula.

\section{Análisis final}

No todas las combinaciones resultan en los efectos esperados y hay casos en los que, a pesar de que hay elementos que por sí solos nos ofrecen importantes ventajas, al unirlos con otros pierden su efectividad. Sin embargo, no es lo que ha ocurrido en nuestro caso, ya que la unión de las TRIC y el modelo del AC nos han ofrecido una hibridación de lo más inclusiva. El motivo del éxito ha sido la complementariedad que existe entre ambos conceptos.

Cuando se implementa el AC como forma de trabajo, se necesita de un constante diálogo entre sus miembros, lo cual es potenciado por la integración de las TRIC. No solo se trata de un medio para iniciar un diálogo en persona, también ofrece al alumnado una vía más para 
comunicarse con el resto de compañeros en la que no es necesario que haya presencia in situ, lo que favorece el trabajo (García y González, 2006). Además, la complejidad que pueden llegar a adquirir determinados programas digitales, requiere de diversas habilidades que no suele tener un mismo individuo, provocando que haya interdependencia positiva entre los estudiantes y exista una mayor igualdad de oportunidades de éxito dentro del grupo (Calzadilla, 2002).

\section{Conclusiones}

Tanto, el modelo del AC como las TRIC nos ofrecen destacables beneficios que nos llevan a lograr prácticas de aula inclusivas. Por separado, el AC se decanta como una metodología a tener en cuenta por su capacidad para maximizar la participación de todos los estudiantes. Pero no hay que olvidar la aparición de elementos, como la tutorización entre iguales o "niño tutor", dentro de cada grupo de trabajo con el que se busca apoyar la participación de los "alumnos excepcionales" (Ainscow, 2012). La aplicación de las TRIC favorece que todo el alumnado se encuentre integrado al favorecer situaciones de diálogo entre los estudiantes en el aula y por ofrecer la posibilidad de hacerlo fuera de ella, a través de soportes digitales. Pero, el verdadero potencial lo encontramos al unir ambos en la propuesta de trabajo que hemos llevado a cabo, donde las ventajas que nos ofrecen por separado se complementan a la perfección. Por tanto, el trabajo se puede resumir con la firme idea de que tanto las TRIC como el AC, por individual, nos permiten llegar hacia una educación mucho más integradora y su hibridación nos ofrece una propuesta de aula inclusiva.

A pesar de las conclusiones expuestas, debemos de tener presente que ni las TRIC, ni el AC son los factores más determinantes para lograr prácticas inclusivas. Cualquier docente que quiera llevar a cabo tales prácticas educativas debe poseer unos conocimientos lo suficientemente asentados como para garantizar la efectividad de sus prácticas. En el caso de las TRIC, es cierto que tienen la capacidad de influir sobre la persona, cambiando su forma de aprender, trabajar o de relacionarse, pero sin un correcto trabajo de aula no se lograrán nuestras pretensiones. La mejora de la práctica educativa no se debe tanto a la inclusión de estos elementos, sino a la forma en la que son utilizados en el proceso de aprendizaje, por alumnado y profesorado (Bustos y Coll, 2010). 
En este sentido, nos encontramos en la obligación de denunciar la falta de formación de aquellos docentes que han visto como su metodología no ha cambiado en los últimos años. Las TIC no solo pueden ser un medio para la educación, sino que tienen la capacidad suficiente como para llegar a convertirse en un fin. Con esta expresión, queremos manifestar que estos medios tecnológicos no solo son simples recursos educativos que se utilizan para apoyar una explicación o hacer alguna actividad puntual. Esas "nuevas tecnologías" a las que posiblemente, se refiera este perfil de docente ya no son tan novedosas. Por lo tanto, dejemos atrás esa denominación, con sus correspondientes prácticas primitivas, para el beneficio del educando. La solución pasa por una mejora en la formación, relacionada con este ámbito. Esta favorecerá un cambio de mentalidad y nos llevará a verlas como un elemento de carácter permanente en la enseñanza. Pues son muchos más los beneficios que ser meramente un complemento. La propuesta de intervención planteada puede convertirse en el ejemplo que abra el camino hacia el cambio sobre su empleo y uso en el aula. Se ha mostrado como su acción, junto a la del modelo del AC, nos permite llegar a objetivos más profundos, como el principio de inclusión educativa, algo que no es para nada sencillo.

Dentro de un aula el denominador común es la diversidad, empezando por el profesor y acabando en su alumnado. Quizás la falta de convicción de que todos somos diferentes sea el mayor obstáculo al que debe enfrentarse la educación inclusiva. El asumir que todos, tanto los catalogados como los que no, tienen necesidades educativas, debería de ser el primer paso para llegar a la inclusión. Algo que puede sonar fácil de decir, pero difícil de pensar. Coincidimos en esta idea con Corona, Peñaloza y Vargas (2017), quiénes exponen que: "la inclusión implica la aceptación y puesta en juego de la diversidad humana y social" ( $p$. 210). Al partir del principio de diversidad humana nadie debería sentirse excluido del sistema educativo por "ser diferente", puesto que se asume que todos lo somos. De modo que, cuando se emplea el término "atención a la diversidad", nos referiremos a las acciones destinadas a satisfacer las necesidades y las diferencias de todo el conjunto de estudiantes que hay en el aula, y no solo a las enfocadas a aquellos grupos estudiantiles que tienen unas peculiaridades tales que requieren de un diagnóstico y un trabajo con profesionales especializados. Queda mucho por trabajar en este sentido, pero como nos muestra el modelo del 
aprendizaje cooperativo, el trabajo en equipo nos ayudará a lograr el objetivo común.

Esta metodología es sumamente interesante para ser implementada en cualquier etapa educativa, pero que se hace más necesaria en edades en las que se está construyendo la personalidad del individuo. El AC fomenta una serie de valores, como son el respeto, la igualdad, la tolerancia, la responsabilidad, la cooperación o la solidaridad, que son los que nos llevarán a construir una sociedad más acogedora en la que todos nos sintamos incluidos en ella. Ese, será el momento en el que este tipo de prácticas ya no sean catalogadas como propuestas de innovación educativa, en el sentido de que la inclusión educativa será una realidad y, por ello, la educación inclusiva dejará de ser necesaria.

Por último, se considera este trabajo una aportación a estudios más amplios sobre AC (Pujolàs, 2004, 2009, 2012), TRIC (Garrido et al., 2016; Marta y Gabelas, 2016) y educación inclusiva (Ainscow, 2012; Arnáiz, 2003; Martínez y Gómez, 2013).

\section{Referencias}

Ainscow, M. (2012). Haciendo que las escuelas sean más inclusivas: lecciones a partir del análisis de la investigación internacional. Revista de Educación Inclusiva, 5(1), 39-49. Recuperado de https://dialnet.unirioja.es/servlet/articulo?codigo $=4105297$

Arnáiz, P. (2003). Educación inclusiva. Una escuela para todos. Málaga: Aljibe.

Barrio, J. L. (2008). Hacia una educación inclusiva para todos. Revista Complutense de Educación, 20(1), 13-31. Recuperado de http://revistas.ucm.es/index.php/RCED/article/view/ RCED0909120013A

Busquet, J., Medina, A. y Ballano, S. (2013). El uso de las TRIC y el choque cultural en la escuela: encuentros y desencuentros entre maestros y alumnos. Revista Mediterránea de Comunicación, 4(2), 115-135. doi: https://doi.org/10.14198/MEDCOM2013.4.2.06

Bustos, A. y Coll, C. (2010). Los entornos virtuales como espacios de enseñanza y aprendizaje. Una perspectiva psicoeducativa para su caracterización y análisis. Revista Mexicana de Investigación Educativa, 15(44), 163-184. Recuperado de http://www.scielo.org.mx/ scielo.php?script=sci_arttext\&pid=S1405-66662010000100009 
Caldevilla, D. (2010). Las redes sociales. Tipología, uso y consumo de las redes 2.0 en la sociedad digital actual. Documentación de las Ciencias de la Información, 33, 45-68. Recuperado de http:// revistas.ucm.es/index.php/DCIN/article/view/dcin1010110045a

Calzadilla, M. E. (2002). Aprendizaje colaborativo y tecnologías de la información y la comunicación. Revista Iberoamericana de Educación, 29(1), 1-10. Recuperado de https://rieoei.org/RIE/article/ view/2868

Corona, F. Y., Peñaloza, I. y Vargas, M. L. (2017). La inclusión de niños con discapacidad intelectual y en situación de calle: una mirada comparativa entre Chile, Colombia y México. Revista Ensayos Pedagógicos, 12(2), 195-215. doi: https://doi.org/10.15359/ rep.12-2.9

Díaz, J. y Troyano, Y. (2013). El potencial de la gamificación aplicado al ámbito educativo. III Jornadas de Innovación Docente. Innovación educativa: respuesta en tiempos de incertidumbre. Universidad de Sevilla: Facultad de Ciencias de la Educación, Sevilla.

Domingo, M. y Marqués, P. (2011). Aulas 2.0 y uso de las TIC en la práctica docente. Comunicar: Revista Cientifica Iberoamericana de Comunicación y Educación, 37, 169-175. Recuperado de https://dialnet.unirioja.es/servlet/articulo? codigo $=3734127$

Fernández, J. (2018). Participación equitativa e igualdad de oportunidades de éxito: sexto y séptimo elementos básicos del aprendizaje cooperativo. XI Congreso Internacional de Actividades Físicas Cooperativas. Ediciones de la Universidad de Oviedo, Avilés.

Ferro, C., Martínez, A. I. y Otero, M. C. (2009). Ventajas del uso de las TICs en el proceso de enseñanza-aprendizaje desde la óptica de los docentes universitarios españoles. Revista Electrónica de Tecnología Educativa, 29, 1-12. doi: https://doi.org/10.21556/ edutec.2009.29.451

García, A. y González, L. (2006). Uso pedagógico de materiales y recursos educativos de las tics: sus ventajas en el aula. II Congreso TIC en Educación. Universidad de Salamanca, Valladolid.

García, R. (2018). El juego para el desarrollo. Una propuesta de intervención en Educación primaria (trabajo fin de grado). Universidad de Valladolid, Segovia, España. Recuperado de http:// uvadoc.uva.es/handle/10324/30469 
Garrido, M., Munté, R. A. y Busquet, J. (2016). De las TIC a las TRIC estudio sobre el uso de las TIC y la brecha digital entre adultos y adolescentes en España. Anàlisi, 54, 44-57. doi: https://doi.org/10.7238/a.v0i54.2953

Gil, J. y Marfil, R. (2018). El empoderamiento del alumnado a través de las TRIC. Creaciones narrativas a través de Stop Motion en educación primaria. Index comunicación, 8(2), 189-210. Recuperado de http://hdl.handle.net/10481/51333

Gutiérrez, A. y Tyner, K. (2012). Educación para los medios, alfabetización mediática y competencia digital. Comunicar, 19(38), 31-39. doi: https://doi.org/10.3916/C38-2012-02-03

Johnson, D. W., Johnson, R. T. y Holubec, E. J. (1999). El aprendizaje cooperativo en el aula. Buenos Aires: Paidós.

Johnson, D. W. y Johnson, R. T. (2014). Cooperative learning in the $21^{\text {st }}$ century. Anales de Psicología, 30(3), 841-851. Recuperado de $\mathrm{http}: / /$ revistas.um.es/analesps/article/view/201241

Kozulin, A. (2000). Instrumentos psicológicos. La educación desde una perspectiva sociocultural. Barcelona: Paidós.

Lozano, R. (2011). De las TIC a las TAC: tecnologías del aprendizaje y del conocimiento. Anuario ThinkEPI, 5, 45-47. Recuperado de https://recyt.fecyt.es/index.php/ThinkEPI/article/view/30465

Marta, C. y Gabelas, J. A. (2016). Comunicación digital. Un modelo basado en el Factor R-elacional. Barcelona: Editorial UOC.

Martínez, A. y Gómez, J. L. (2013). Escuelas inclusivas singulares. Madrid: Editorial Grupo 5.

Martínez, P. C. (2006). El método de estudio de caso. Estrategia metodológica de la investigación científica. Revista científica Pensamiento y Gestión, 20, 165-193. Recuperado de http:// rcientificas.uninorte.edu.co/index.php/pensamiento/article/ viewFile/3576/2301

Monge, C. y Gómez, P. (2018). Implicaciones de la formación e innovación en la mejora de la calidad educativa. En C. Monge, y P. Gómez (Eds.), Innovando la docencia desde la formación del profesorado. Madrid: Síntesis.

Osuna, S. y Gil, J. (2017). El proyecto europeo ECO. Rompiendo barreras en el acceso al conocimiento. Educación XX1, 20(2), 189213. doi: https://doi.org/10.5944/educxx1.19037 
Osuna, S., Gil, J. y Cantillo, C. (2018). La construcción de la identidad infantil en el Mundo Disney. Revista Latina de Comunicación Social, 73, 1284-1307.doi:https://doi.org/10.4185/ RLCS-2018-1307

Pujolàs, P. (2004). Aprender juntos alumnos diferentes. Los equipos de aprendizaje cooperativo en el aula. Barcelona: Octaedro-Eumo.

Pujolàs, P., Riera, G., Pedragosa, O. y Soldevila, J. (2005). Aprender juntos alumnos diferentes (I) El "qué" y el "cómo" del aprendizaje cooperativo en el aula. España: Octaedro.

Pujolàs, P. (2009). El aprendizaje cooperativo. 9 ideas clave. Barcelona: Graó.

Pujolàs, P. (2012). Aulas inclusivas y aprendizaje cooperativo. Educatio Siglo XXI, 30(1), 89-112. Recuperado de http://repositori. uvic.cat/handle/10854/1998

Rodríguez, E. (2009). Ventajas e inconvenientes de las TICs en el aula. Cuaderno de Educación y Desarrollo, 1 (9), 1. Recuperado de https://ideas.repec.org/a/erv/cedced/y2009i914.html

Slavin, R. E. y Johnson, R. T. (1999). Aprendizaje cooperativo: teoría, investigación y práctica. Buenos Aires: Aique.

Traver, J. A. y García, R. (2006). La técnica puzzle de Aronson como herramienta para desarrollar la competencia «compromiso ético» y la solidaridad en la enseñanza universitaria. Revista Iberoamericana de Educación, 40(4), 1-9. Recuperado de https://rieoei. org/RIE/article/view/2499

Velázquez, C., Fraile, A. y López, V. M. (2014). Aprendizaje cooperativo en educación física. Movimento. Revista da Escola de Educação Física, 20(1), 239-259. Recuperado de https://seer.ufrgs. br/index.php/Movimento/article/view/40518/28352 